General Mathematics Vol. 28, No. 1 (2020), 59-66

DOI:10.2478/gm-2020-0005

S sciendo

\title{
Calculus for the intermediate point associated with a mean value theorem of the integral calculus ${ }^{1}$
}

\author{
Emilia-Loredana Pop, Dorel Duca, Augusta Raţiu
}

\begin{abstract}
If $f, g:[a, b] \rightarrow \mathbb{R}$ are two continuous functions, then there exists a point $c \in(a, b)$ such that

$$
\int_{a}^{c} f(x) d x+(c-a) g(c)=\int_{c}^{b} g(x) d x+(b-c) f(c) .
$$

In this paper, we study the approaching of the point $c$ towards $a$, when $b$ approaches $a$.
\end{abstract}

2010 Mathematics Subject Classification: 26A24, 11H60.

Key words and phrases: intermediate point, mean-value theorem.

\section{Introduction and Preliminaries}

Let $a, b \in \mathbb{R}$ with $a<b$ and $f, g:[a, b] \rightarrow \mathbb{R}$ two continuous functions. By Rolle's theorem (see, for example [1], [2]), applied to the function $h:[a, b] \rightarrow \mathbb{R}$ defined by

$$
h(x)=(b-x) \int_{a}^{x} f(t) d t+(x-a) \int_{x}^{b} g(t) d t, \text { for all } x \in[a, b],
$$

there exists a point $c \in(a, b)$ such that

$$
\int_{a}^{c} f(x) d x+(c-a) g(c)=\int_{c}^{b} g(x) d x+(b-c) f(c) .
$$

\footnotetext{
${ }^{1}$ Received 8 January, 2020

Accepted for publication (in revised form) 27 May, 2020
} 
It follows that, for each $x \in(a, b]$, there exists a point $c_{x} \in(a, x)$ such that

$$
\int_{a}^{c_{x}} f(t) d t+\left(c_{x}-a\right) g\left(c_{x}\right)=\int_{c_{x}}^{x} g(t) d t+\left(x-c_{x}\right) f\left(c_{x}\right) .
$$

If for each $x \in(a, b]$ we choose one $c_{x} \in(a, x)$ such that (1) holds, we can define the function $c:(a, b] \rightarrow[a, b]$ by

$$
c(x)=c_{x} \text {, for all } x \in(a, b],
$$

with the property

$$
\begin{aligned}
& \int_{a}^{c(x)} f(t) d t+(c(x)-a) g(c(x))=\int_{c(x)}^{x} g(t) d t+(x-c(x)) f(c(x)), \\
& \quad \text { for all } x \in(a, b] .
\end{aligned}
$$

So, we have that

Theorem 1 Let $a, b \in \mathbb{R}$ with $a<b$. If the functions $f, g:[a, b] \rightarrow \mathbb{R}$ are continuous, then there exists a function $c:(a, b] \rightarrow[a, b]$ such that (3) holds.

When $x \in(a, b]$ tends to $a$, because $|c(x)-a| \leq|x-a|$, we have

$$
\lim _{x \rightarrow a} c(x)=a .
$$

Then the function $\bar{c}:[a, b] \rightarrow[a, b]$ defined by

$$
\bar{c}(x)= \begin{cases}c(x), & \text { if } x \in(a, b] \\ a, & \text { if } x=a .\end{cases}
$$

is continuous at $x=a$.

For $x \in(a, b]$, we have

$$
\frac{\bar{c}(x)-\bar{c}(a)}{x-a}=\frac{c(x)-a}{x-a} .
$$

If we denote by

$$
\theta(x)=\frac{c(x)-a}{x-a}
$$

then

$$
\theta(x) \in(0,1]
$$

and

$$
c(x)=a+(x-a) \theta(x)
$$

and hence

$$
\begin{aligned}
& \int_{a}^{a+(x-a) \theta(x)} f(t) d t+(x-a) \theta(x) g(a+(x-a) \theta(x))= \\
& =\int_{a+(x-a) \theta(x)}^{x} g(t) d t+(x-a)(1-\theta(x)) f(a+(x-a) \theta(x)), \\
& \text { for all } x \in(a, b] .
\end{aligned}
$$

Hence, the following result is true. 
Theorem 2 Let $a, b \in \mathbb{R}$ with $a<b$. If the functions $f, g:[a, b] \rightarrow \mathbb{R}$ are continuous, then there exists a function $\theta:(a, b] \rightarrow(0,1]$ such that $(4)$ holds.

If $F$ is a primitive of the function $f$ and $G$ is a primitive of the function $g$, then, from the Leibniz-Newton Theorem we have

$$
\begin{gathered}
\int_{a}^{a+(x-a) \theta(x)} f(t) d t=F(a+(x-a) \theta(x))-F(a), \\
\int_{a+(x-a) \theta(x)}^{x} g(t) d t=G(x)-G(a+(x-a) \theta(x))
\end{gathered}
$$

and the equality (4) can be written as

$$
\begin{aligned}
& F(a+(x-a) \theta(x))-F(a)+g(a+(x-a) \theta(x)) \theta(x)(x-a)= \\
& =G(x)-G(a+(x-a) \theta(x))+f(a+(x-a) \theta(x))(1-\theta(x))(x-a), \\
& \text { for all } x \in(a, b] .
\end{aligned}
$$

The purpose of this paper is to establish under which circumstances the function $\bar{c}$ is differentiable at the point $x=a$ and to compute its derivatives.

The idea was presented for different approaches, by using other hypothesis and obtaining corresponding results for other interesting problems, like the ones presented in [3], [4], [5], [6], [7], [8].

\section{Main results}

Theorem 3 Let $a, b \in \mathbb{R}$ with $a<b$ and let $f, g$ be two differentiable functions on $[a, b]$. If $f(a)+g(a) \neq 0$, then:

$1^{\circ}$ The function $\theta$ has the property that

$$
\lim _{x \rightarrow a} \theta(x)=\frac{1}{2} .
$$

$2^{\circ}$ The function $\bar{c}$ is differentiable at $x=a$ and

$$
\bar{c}^{\prime}(a)=\frac{1}{2} .
$$

Proof. We consider relation (5) and we divide it by $(x-a)$ and get

$$
\begin{aligned}
& \frac{F(a+(x-a) \theta(x))-F(a)}{x-a}+g(a+(x-a) \theta(x)) \theta(x)= \\
& =\frac{G(x)-G(a+(x-a) \theta(x))}{x-a}+f(a+(x-a) \theta(x))(1-\theta(x)),
\end{aligned}
$$

for all $x \in(a, b]$. 
Next, we adjust the relation with $\theta(x)$ and manage $G(a)$, such that

$$
\begin{aligned}
& \frac{F(a+(x-a) \theta(x))-F(a)}{(x-a) \theta(x)} \theta(x)+g(a+(x-a) \theta(x)) \theta(x)=\frac{G(x)-G(a)}{x-a}+ \\
& +\frac{G(a)-G(a+(x-a) \theta(x))}{(x-a) \theta(x)} \theta(x)+f(a+(x-a) \theta(x))(1-\theta(x)), \\
& \text { for all } x \in(a, b] .
\end{aligned}
$$

When we pass to $\lim _{x \rightarrow a}$, we get

$$
F^{\prime}(a) \lim _{x \rightarrow a} \theta(x)+g(a) \lim _{x \rightarrow a} \theta(x)=G^{\prime}(a)-G^{\prime}(a) \lim _{x \rightarrow a} \theta(x)+f(a)\left(1-\lim _{x \rightarrow a} \theta(x)\right) .
$$

But $F^{\prime}=f$ ad $G^{\prime}=g$. Consequently, we have

$$
f(a) \lim _{x \rightarrow a} \theta(x)+g(a) \lim _{x \rightarrow a} \theta(x)=g(a)-g(a) \lim _{x \rightarrow a} \theta(x)+f(a)\left(1-\lim _{x \rightarrow a} \theta(x)\right) .
$$

But $f(a)+g(a) \neq 0$ and hence $2 \lim _{x \rightarrow a} \theta(x)=1$ and so,

$$
\lim _{x \rightarrow a} \theta(x)=\frac{1}{2} \text { and } \bar{c}^{\prime}(a)=\frac{1}{2} .
$$

Theorem 4 Let $a, b \in \mathbb{R}$ with $a<b$ and let $f, g$ be two times differentiable functions on $[a, b]$. If $f(a)+g(a) \neq 0$, then

$1^{\circ}$ The function $\bar{\theta}:[a, b] \rightarrow[0,1]$ defined by

$$
\bar{\theta}(x)= \begin{cases}\theta(x), & \text { if } x \in(a, b] \\ \frac{1}{2}, & \text { if } x=a\end{cases}
$$

is differentiable at $x=a$ and

$$
\bar{\theta}^{\prime}(a)=\frac{f^{\prime}(a)+g^{\prime}(a)}{8[f(a)+g(a)]} .
$$

$2^{\circ}$ The function $\bar{c}$ is two times differentiable at $x=a$ and

$$
\bar{c}^{\prime \prime}(a)=\frac{f^{\prime}(a)+g^{\prime}(a)}{4[f(a)+g(a)]} .
$$

Proof. First, we rewrite, relation (6) in the following form: (7)

$$
\begin{aligned}
& \frac{F(a+(x-a) \theta(x))-F(a)}{(x-a) \theta(x)} \theta(x)+\frac{G(a+(x-a) \theta(x))-G(a)}{(x-a) \theta(x)} \theta(x)+g(a+(x-a) \theta(x)) \theta(x)+ \\
& +f(a+(x-a) \theta(x)) \theta(x)=\frac{G(x)-G(a)}{x-a}+f(a+(x-a) \theta(x)), \\
& \text { for all } x \in(a, b] .
\end{aligned}
$$


We construct $\frac{\bar{\theta}(x)-\bar{\theta}(a)}{x-a}=\frac{\theta(x)-\frac{1}{2}}{x-a}$. From relation (7) we have

$$
\begin{aligned}
& \frac{F(a+(x-a) \theta(x))-F(a)}{(x-a) \theta(x)}\left(\theta(x)-\frac{1}{2}\right)+\frac{1}{2} \frac{F(a+(x-a) \theta(x))-F(a)}{(x-a) \theta(x)}+ \\
& +\frac{G(a+(x-a) \theta(x))-G(a)}{(x-a) \theta(x)}\left(\theta(x)-\frac{1}{2}\right)+\frac{1}{2} \frac{G(a+(x-a) \theta(x))-G(a)}{(x-a) \theta(x)}+ \\
& +\left(\theta(x)-\frac{1}{2}\right) g(a+(x-a) \theta(x))+\frac{1}{2} g(a+(x-a) \theta(x))+ \\
& +\left(\theta(x)-\frac{1}{2}\right) f(a+(x-a) \theta(x))+\frac{1}{2} f(a+(x-a) \theta(x))= \\
& =\frac{G(x)-G(a)}{x-a}+f(a+(x-a) \theta(x)), \text { for all } x \in(a, b] .
\end{aligned}
$$

We divide this relation by $(x-a)$ and we get

$$
\begin{aligned}
& \frac{F(a+(x-a) \theta(x))-F(a)}{(x-a) \theta(x)} \frac{\theta(x)-\frac{1}{2}}{x-a}+\frac{1}{2} \frac{F(a+(x-a) \theta(x))-F(a)}{(x-a)^{2} \theta(x)}+ \\
& +\frac{G(a+(x-a) \theta(x))-G(a)}{(x-a) \theta(x)} \frac{\theta(x)-\frac{1}{2}}{x-a}+\frac{1}{2} \frac{G(a+(x-a) \theta(x))-G(a)}{(x-a)^{2} \theta(x)}+ \\
& +\frac{\theta(x)-\frac{1}{2}}{x-a} g(a+(x-a) \theta(x))+\frac{1}{2(x-a)} g(a+(x-a) \theta(x))+ \\
& +\frac{\theta(x)-\frac{1}{2}}{x-a} f(a+(x-a) \theta(x))+\frac{1}{2(x-a)} f(a+(x-a) \theta(x))= \\
& =\frac{G(x)-G(a)}{(x-a)^{2}}+\frac{f(a+(x-a) \theta(x))}{x-a}, \\
& \text { for all } x \in(a, b] .
\end{aligned}
$$

We pass to $\lim _{x \rightarrow a}$ in relation (9) and we obtain

$$
\bar{\theta}^{\prime}(a)[f(a)+g(a)]=I,
$$

where

$$
\begin{aligned}
& I=\lim _{x \rightarrow a}\left[\frac{G(x)-G(a)}{(x-a)^{2}}-\frac{1}{2} \frac{F(a+(x-a) \theta(x))-F(a)}{(x-a)^{2} \theta(x)}-\frac{1}{2} \frac{G(a+(x-a) \theta(x))-G(a)}{(x-a)^{2} \theta(x)}+\right. \\
& \left.+\frac{f(a+(x-a) \theta(x))}{2(x-a)}-\frac{g(a+(x-a) \theta(x))}{2(x-a)}\right]
\end{aligned}
$$

We separate this limit in 2 parts $I=I_{1}+I_{2}$ and we have:

$$
\begin{aligned}
I_{1} & =\frac{-1}{2} \lim _{x \rightarrow a}\left[\frac{F(a+(x-a) \theta(x))-F(a)}{(x-a)^{2} \theta(x)}-\frac{f(a+(x-a) \theta(x))}{x-a}\right]= \\
& =\frac{-1}{2} \lim _{x \rightarrow a}\left[\frac{\frac{F(a+(x-a) \theta(x))-F(a)}{(x-a) \theta(x)}-f(a+(x-a) \theta(x))}{(x-a)}\right]=
\end{aligned}
$$




$$
\begin{gathered}
=\frac{-1}{2} \lim _{x \rightarrow a}\left[\frac{\frac{F(a+(x-a) \theta(x))-F(a)}{(x-a) \theta(x)}-F^{\prime}(a)}{(x-a)}-\frac{f(a+(x-a) \theta(x))-f(a)}{x-a}\right]= \\
=\frac{-1}{2} \lim _{x \rightarrow a} \theta(x)\left[\frac{\frac{F(a+(x-a) \theta(x))-F(a)}{(x-a) \theta(x)}-F^{\prime}(a)}{(x-a) \theta(x)}-\frac{f(a+(x-a) \theta(x))-f(a)}{(x-a) \theta(x)}\right] . \\
=\frac{-1}{4}\left[\frac{F^{\prime \prime}(a)}{2}-f^{\prime}(a)\right]=\frac{f^{\prime}(a)}{8} .
\end{gathered}
$$

and

$$
\begin{aligned}
& I_{2}=\lim _{x \rightarrow a}\left[\frac{G(x)-G(a)}{(x-a)^{2}}-\frac{G(a+(x-a) \theta(x))-G(a)}{2(x-a)^{2} \theta(x)}-\frac{g(a+(x-a) \theta(x))}{2(x-a)}\right]= \\
& =\frac{-1}{2} \lim _{x \rightarrow a}\left[\frac{\frac{G(a+(x-a) \theta(x))-G(a)}{(x-a) \theta(x)}+g(a+(x-a) \theta(x))-2 \frac{G(x)-G(a)}{x-a}}{(x-a)}\right]= \\
& =\frac{-1}{2} \lim _{x \rightarrow a}\left[\frac{\frac{G(a+(x-a) \theta(x))-G(a)}{(x-a) \theta(x)}-G^{\prime}(a)}{(x-a)}+\frac{G^{\prime}(a)+g(a+(x-a) \theta(x))-2 \frac{G(x)-G(a)}{x-a}}{x-a}\right]= \\
& =\frac{-1}{2} \lim _{x \rightarrow a} \frac{\frac{G(a+(x-a) \theta(x))-G(a)}{(x-a) \theta(x)}-G^{\prime}(a)}{\theta(x)(x-a)} \theta(x)+ \\
& +\lim _{x \rightarrow a} \frac{\frac{G(x)-G(a)}{x-a}-G^{\prime}(a)}{x-a}-\frac{1}{2} \lim _{x \rightarrow a} \frac{g(a+(x-a) \theta(x))-g(a)}{\theta(x)(x-a)} \theta(x)= \\
& =-\frac{G^{\prime \prime}(a)}{4} \theta(a)+\frac{G^{\prime \prime}(a)}{2}-\frac{g^{\prime}(a)}{2} \theta(a)=\frac{g^{\prime}(a)}{8} \text {. }
\end{aligned}
$$

From

$$
I=I_{1}+I_{2},
$$

we obtain

$$
\bar{\theta}^{\prime}(a)[f(a)+g(a)]=\frac{f^{\prime}(a)+g^{\prime}(a)}{8} .
$$

Hence

$$
\bar{\theta}^{\prime}(a)=\frac{f^{\prime}(a)+g^{\prime}(a)}{8[f(a)+g(a)]}
$$

and

$$
\bar{c}^{\prime \prime}(a)=\frac{f^{\prime}(a)+g^{\prime}(a)}{4[f(a)+g(a)]} .
$$




\section{Conclusions and further challenges}

In this paper we introduced conditions for the functions $f$ and $g$ such that the intermediate point of functions $\bar{c}$ and $\bar{\theta}$ to be derivable at the point $a$ and we provided the derivative $\bar{\theta}^{\prime}(a), \bar{c}^{\prime}(a)$ and $\bar{c}^{\prime \prime}(a)$.

In future we want to see in which conditions the functions $\bar{c}$ and $\bar{\theta}$ are derivable of order $n$ at the point $a$ and to calculate the corresponding derivatives.

\section{References}

[1] D. I. Duca, Analiză Matematică, Casa Cărţii de Ştiinţăa, Cluj-Napoca, vol. I, 2013.

[2] D. I. Duca, Analiză Matematică, vol. II, to appear.

[3] D. I. Duca, Properties of the intermediate point from the Taylor's theorem, Mathematical Inequalities and Applications, vol. 12, no. 4, 763-771, 2009.

[4] D. I. Duca, E. Duca, Exerciţii şi probleme de analiză matematică, Casa Cărţii de Ştiinţă, vol. II, 2009 .

[5] D. I. Duca, O. Pop, Concerning the Intermediate Point in the Mean-Value Theorem, Mathematical Inequalities \& Applications, vol. 12, vol. 3, 499-512, 2009.

[6] E. L. Pop, D. I. Duca, A. Ratiu, Properties of the intermediate point from a mean value theorem of the integral calculus, General Mathematics, vol. 25, no.1-2, 2017, 3-10.

[7] E. L. Pop, D. I. Duca, A. Ratiu, Properties of the intermediate point from a mean value theorem of the integral calculus - II, General Mathematics, vol. 27, no. 1, 2019, 29-39.

[8] T. Trif, Asymptotic Behavior of Intermediate Points in certain Mean Value Theorems, Journal of Mathematical Inequalities, vol. 2, no. 2, 2008, 151-161.

\section{Emilia-Loredana Pop}

Babeş-Bolyai University

Faculty of Mathematics and Computer Science

Department of Computer Science

No. 1 Mihail Kogalniceanu Street, RO-400084 Cluj-Napoca, Romania

e-mail: pop_emilia_loredana@yahoo.com 


\section{Dorel Duca}

Babeş-Bolyai University

Faculty of Mathematics and Computer Science

Department of Mathematics

No. 1 Mihail Kogalniceanu Street, RO-400084 Cluj-Napoca, Romania

e-mail: dorelduca@yahoo.com

\section{Augusta Raţiu}

Lucian Blaga University of Sibiu

Faculty of Science

Department of Mathematics and Computer Science

No. 5-7 Dr. I. Raţiu Street, RO-550012 Sibiu, Romania

e-mail: augu2003@yahoo.com 